\title{
EVALUATION OF VESTIBULAR FUNCTION IN CHILDREN WITH PURULENT MEDIA OTITIS BY COMPUTER STABILOMETRY
}

\author{
Iuliia Lozova $₫$ \\ Department of Otorhinolaryngology and Pediatric Otolaryngology ${ }^{l}$ \\ Children's Department ${ }^{2}$ \\ ukrlor@gmail.com \\ Grigoriy Garyuk \\ Department of Otorhinolaryngology and Pediatric Otolaryngology ${ }^{l}$ \\ Tatiana Pochuieva \\ Department of Otorhinolaryngology and Pediatric Otolaryngology ${ }^{1}$ \\ Iryna Redka \\ Department of Hygiene and Social Medicine \\ V. N. Karazin Kharkiv National University \\ 4 Svobody sq., Kharkiv, Ukraine, 61022
}

Serhii Samusenko ${ }^{2}$

${ }^{1}$ Kharkiv Academy of Postgraduate Education

58 Amosova str., Kharkiv, Ukraine, 61176

${ }^{2}$ Kharkiv city hospital No. 30

5/7 Gudanova str., Kharkiv, Ukraine, 61024

$\bowtie$ Corresponding author

\begin{abstract}
The aim of the research - assessment of vestibular function in children with acute purulent otitis media by computer static stabilometry compared with typically developed children of the same age.

Materials and methods: 22 children with purulent otitis media aged 4 to 7 years and 30 practically healthy peers were examined by static stabilometry on the device «MPFI stabilograph 1» (Kharkiv, Ukraine). Indicators of variation and shape of the pressure center distribution, spectral-correlation indicators of stabilograms, as well as integral indicators of stability (length, velocity and angle of postural oscillations) were taken into account and the equilibrium functions of the two sensory states (open and closed eyes) are calculated in the StabiliS software.

Results: purulent otitis media causes posterior displacement of the absolute coordinates of the pressure center. In the state with closed eyes, children with purulent otitis differ from their healthy coevals by 14 of 24 stabilometric parameters, including stabilogram length, speed, angle and amplitude of oscillations, giving way to them in the stability of equilibrium. Children with purulent otitis media are more sensitive to the visual canal of postural control than their coevals. Involvement of visual afferents in postural control significantly improves balance maintenance in children with purulent otitis by reducing deviations of postural oscillations, which is reflected in the probable changes of 9 out of 24 stabilometric parameters compared to control.

Conclusions: In preschool age, purulent otitis media leads to latent vestibular dysfunction, which is manifested by a violation of the stability of the vertical posture in a state with closed eyes and is compensated by visual-vestibulo-proprioceptive integration in the process of postural control.
\end{abstract}

Keywords: purulent otitis media, posturography, balance, vestibular function, preschool age.

DOI: $10.21303 / 2504-5679.2021 .002172$

\section{Introduction}

Peripheral vestibular disorders are difficult to differentiate due to non-specific symptoms, unclear formulation of patient complaints, multifactorial nature of dizziness and posture instability 
and lack of reliable screening methods [1]. All tests of the peripheral nervous system currently used in clinical practice are based on the measurement of oligosynaptic reflexes of the brain stem [2]. Stabilometry, based on the assessment of human balance, which allows to detect vestibulo-spinal disorders, is no exception [3].

Equilibrium is the ability to maintain the center of pressure of the body with minimal shaking [4], and its control is provided by a combination of central and peripheral nerve components, including spinal reflexes, supraspinal commands and integration of afferent and/or efferent signals of visual, vestibular and somato-sensor. Postural control strategies include either compensatory posture adjustments after an unforeseen violation, or proactive posture adjustments that counteract any predicted posture disorders [5]. Postural control is closely related to the harmonious development of sensory-motor integration and motor, cognitive and social development of the child [6].

Stabilometry, also known as stabilography or posturography, is a test in which the force platform is used to objectively assess the balance and obtain a quantitative approximation of the oscillations of the human mass center by registering the pressure center on the platform $[3,5]$. There are static and dynamic stabilometry.

Static stabilometry refers to the measurement of postural oscillations of the center of pressure of the body during a quiet standing on a stationary support surface. Static posturography allows you to objectively assess the passive control of posture [5, 7], which includes the reaction to gravity and the effects of relatively small, self-initiated corrective movements [7]. Dynamic posturography involves the use of induced imbalance, such as displacement of the support surface using an unstable support surface, movement of the visual environment, application of stimuli to the upper body or arbitrary displacement of the center of mass [5]. Simulation of different sensory states allows to evaluate different mechanisms of translational control. Thus, a neutral position on a stable solid surface facing forward with open eyes allows us to assess the integrative interaction of the visual, somatosensory and vestibular systems in maintaining balance [8], while closing the eyes in the same position can assess the effects of somatosensory and vestibular systems [8], and replacement of a stationary platform with a moving one with closed eyes allows to assess mainly vestibular functions, and a solid surface with a pliable one - mainly somatosensory function [2].

Previous studies have demonstrated the effectiveness of postural tests in detecting subclinical vestibular dysfunction even in patients without a history of dizziness, especially in sensory modelling [8].

In the special literature, little attention is paid to vestibular function in children, probably due to the low prevalence of vestibular disorders in the population of children with normal ontogenetic development. At the same time, the auditory system is closely related to the vestibular system in terms of embryological development, innervation, and blood supply [9]. The connection of middle ear diseases with vestibular peripheral disorders is also known [10, 11]. Thus, in children 10-12 years with a history of tympanostomy tubes and/or 3 or more infectious diseases of the ear, transferred to five years, postural stability was reduced according to the computerized test of dynamic posturography to assess stability (SET) and children's balance scale (PBS) [12].

One of the most common diseases of the middle ear in childhood is acute otitis media, including purulent. Previous studies have shown that $50 \%$ of children suffer from acute purulent otitis media before the age of 1 year, and up to $80 \%$ - up to 3 years [13].

According to the results of our retrospective study [14], children with acute purulent otitis media complained of weakness, headache, dizziness, nausea, sometimes vomiting, and otoneurological examination revealed small-swept horizontal nystagmus, shakiness in the Romberg position, «smearing» when performing coordinating tests. This suggests that the complaints of patients are due not only to the processes of intoxication, but also disorders of vestibular function.

Most of the research in the last decade has focused on vestibular function in children with secretory otitis media. In particular, it has been shown that $50 \%$ of children with serous otitis may have some imbalance [15].

In children 3-6 years with moderate secretory otitis, the average rate of oscillation of the body according to computer stabilometry is disturbed both before and after surgery at 3 months, which is considered by the authors as a sign of latent vestibular dysfunction [16]. In the study of 
children 47 years with effusion in the middle ear, no differences were found with the control in the tests of Barany but found not significantly higher rocking speed according to posturography [17].

The study of coordination, balance, oculomotor function and nystagmus in children 4-8 years with otitis media with effusion revealed a significant increase in oculomotor abnormalities compared with the control [18]. A study of 4-15-year-old children with otitis media with effusion and dizziness by video pulse vestibulo-ocular test (vHIT) did not reveal significant changes in the mean values of vHIT growth or their asymmetry, however, revealed an increased frequency of latent saccades (57 \%), which was considered by the authors as a sign of mild vestibular disorder [19]. In another study of the same category of patients by the same method, the frequency of latent saccades (in the vertical planes of the canal) was $32 \%$ and did not depend on the stage of acute secretory otitis with effusion. A significant decrease in vHIT growth was found only in the plane of the anterior canal on the affected side compared to a healthy ear [20]. Posturographic examination of children 4-14 years with bilateral acute otitis externa revealed an imbalance (increase in most stabilometric parameters) both before drainage and after 4 weeks from the moment of drainage [21]. Posturographic examination in combination with electronystagmography (ENG) of children 7-15 years with otitis media with effusion revealed increased values of stabilometric parameters with both open and closed eyes, spontaneous (40.9\%) and positional (63.6\%) nystagmus [22]. In children with otitis media with effusion, the scores on the Arabic dizziness questionnaire and some results of bed tests were significantly deviated from the norm, in $73 \%$ air-induced vestibulo-induced myogenic potentials (oVEMP) had a significant latency delay. At the same time, bone vestibule-induced myogenic potentials (cVEMP) did not differ from control [15].

As we can see, the results of assessment of vestibular function in children with acute otitis media by various instrumental methods are quite contradictory, as indicated in a recent systematic review [11], and studies of vestibular function in children with acute purulent otitis media are virtually absent.

Given the above and the simplicity and reproducibility of the results of static stabilometry in the detection of vestibular dysfunction in children [9], the aim of the study was to assess the state of vestibular function in children with acute purulent otitis media by computer static stabilometry.

\section{Materials and methods}

The study was conducted on the basis of the pediatric otolaryngology department of the «Kharkiv City Hospital No. 30» during 2021. The study was agreed with the ethics committee of the «Kharkiv City Hospital No. 30» (protocol No. 1 from 15.04.2021).

52 children aged 4-7 years were examined, which were divided into 2 groups: the main group ( $n=22$, age $-5.58 \pm 0.24$ years) with purulent acute otitis media and the control group almost healthy children $(n=30$, age $-5.91 \pm 0.21$ years $)$.

Criteria for inclusion in the control group were the absence of acute and chronic pathology of the nervous, vestibular, auditory, visual and musculoskeletal systems.

Criteria for exclusion from the main group were: the presence of pathology of the musculoskeletal and/or visual, nervous systems.

The work was performed in accordance with the Code of Ethics of the World Medical Association (Helsinki Declaration). The work was approved by the Commission on Bioethics of the Kharkiv Medical Academy of Postgraduate Education, protocol No. 3 10.10.2018 Parents or official representatives of children included in the study gave written informed consent to examine children on a stabilometric platform.

Studies of autonomic-spinal reflexes and balance function were performed in the first 2 days of admission to the hospital by the method of computer static stabilometry on the device «MPFI stabilograph 1» (LLC «ASTER IT», Kharkiv, Ukraine). The reliability of stabilometric assessment in children is proven [23].

During the study, the child was on a strain gauge platform $(300 \times 300 \mathrm{~mm})$ in a vertical position in a European rack (heels together, socks apart). The duration of the study according to the recommendations [24] was $1 \mathrm{~min}$. in two sensory states - closed and open eyes. The movement of the pressure center on the platform area was recorded with a sampling frequency of $13.128 \mathrm{~Hz}$.

On the basis of the registered data in the StabiliS software package the following stabilometric indicators were calculated: Length, $\mathrm{mm}$ - length of a trajectory of fluctuations of the pressure center; 
AvgSpeed, $\mathrm{mm} / \mathrm{s}$ - average speed of movement of the pressure center; Angle, ${ }^{\circ}$ is the average angle of oscillation of the pressure center; Pup2Sigma, $\%$ - relative number of stabilogram points lying within the double standard deviation; Pirson $X Y$ - coefficient of linear correlation between oscillations in the frontal and sagittal planes; $w A v g F X Y, H z$ - weighted average frequency of the cross-spectrum of pressure center oscillations in the frontal and sagittal planes; $K F R$, $\%$ - quality of the equilibrium function. The following indicators were calculated separately for the frontal $(X)$ and sagittal $(Y)$ planes: RangeXtiRangeY, mm - amplitude of pressure center oscillations; MeanX and MeanY, mmaverage position of the pressure center; StdDevX and StdDevY, $m m$ - standard deviation of pressure center oscillations; SkewX and SkewY, mm - asymmetry of pressure center oscillations; Kurtosis $X$ and KurtosisY, $\mathrm{mm}$ - excess of pressure center oscillations; wAvgFX and $w A v g F Y, H z$ - weighted average frequency of the spectrum of oscillations of the pressure center; $C C O X$ and $C C O Y$, $s$ is the offset of the autocorrelation function to obtain the value of the correlation coefficient less than zero.

All data were statistically processed in the environment of specialized application software STATISTICA v12.5 (StatSoftInc, USA). The Shapiro-Wilk test was used to check the normality of the distribution. Due to the inconsistency of the law of normal distribution of most of the studied stabilometric indicators, the data are presented in the form of median and interquantile scatter. The Mann-Whitney U-test was used for comparative analysis of the main and control groups, and Wilcoxon's T-test was used to compare different sensory states. Differences at $p \leq 0.05$ were considered significant.

\section{Results}

In the absence of visual afferentation in children with purulent otitis there was a significant $(p \leq 0.001)$ shift of the tic center compared with the control group. Thus, in the control group, the pressure center was almost centered relative to the midline in the frontal plane and slightly displaced backwards, while in purulent otitis there was a more pronounced shift to the right and back relative to the midline (Fig. 1).

A

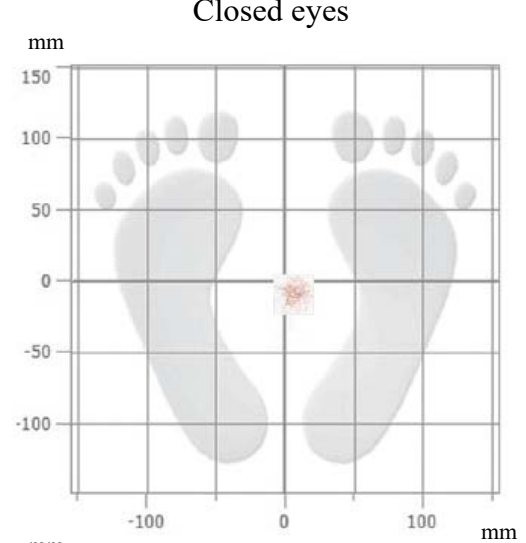

B

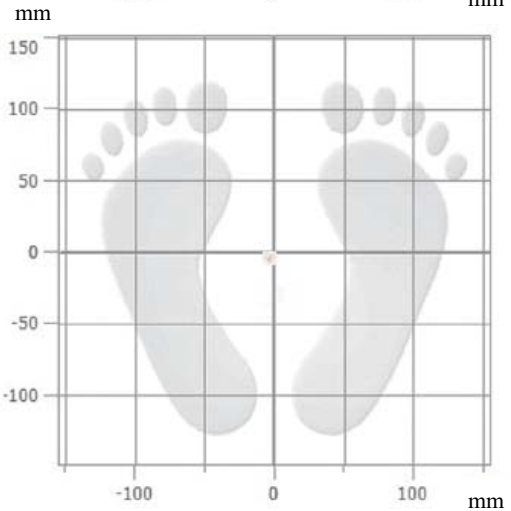

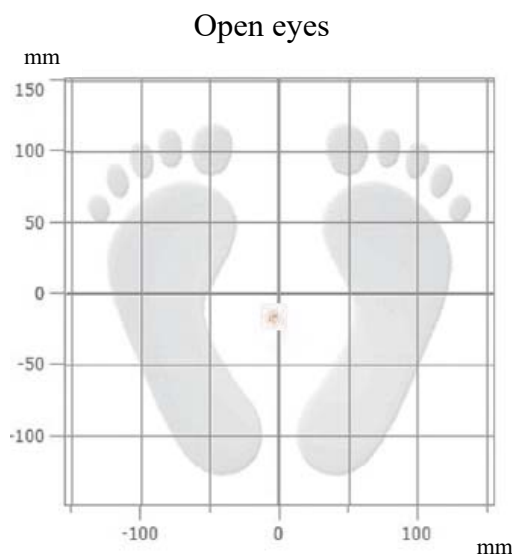

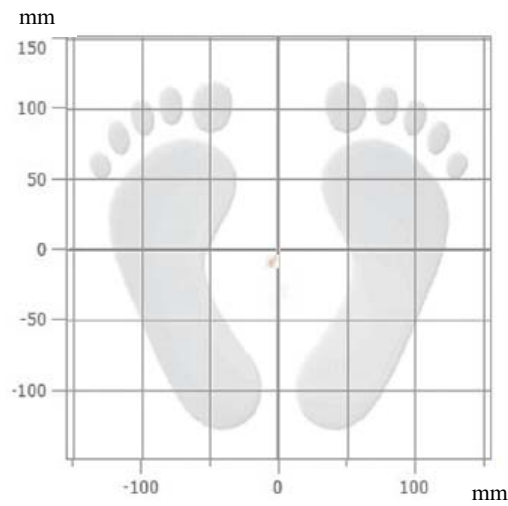

Fig. 1. Example of the position of the pressure center in the coordinate system of the European standard in children with purulent otitis media (A) and in the control (B) under different sensory conditions 
With open eyes, significant changes in the location of the pressure center in children with purulent otitis, compared with controls, were observed only in the sagittal plane $(p \leq 0.05)$, remaining shifted back relative to the midline (Table 1).

Involvement of the visual canal in posturographic control in both groups led to a significant shift of the center of pressure to the left of the midline $(p \leq 0.05)$ in purulent otitis, while in the control there was only a trend $(p=0.090)$.

According to the results of our study, the average figure of the projection of the pressure center in the sample with closed eyes (Fig. 2, A) is actually represented by a circle with purulent otitis media (ratio of $Y / X$ axes $=0.94[0.80 ; 1.11]$ ), and in the control group - ellipse (the ratio of the axes $Y / X=1.12[1.02 ; 1.17])$. This indicates the lack of dominance of the ankle posture support strategy, which regulates stability in the posterior-anterior direction and is typical for the control of purulent otitis (Fig. 2, A) in the absence of visual control.

The median values of the asymmetry coefficients (SkewX, SkewY) by module in both groups did not exceed 0.25 , which indicated a relatively symmetrical position of the pressure center in both planes (Table 2). Under different sensory states, children with purulent otitis media did not differ statistically significantly from the control of the symmetry of the main rack $(p>0.05)$.

Table 1

Coordinates of the position of the pressure center in children with purulent otitis media in different sensory states

\begin{tabular}{ccccc}
\hline \multirow{2}{*}{ Indicators } & \multirow{2}{*}{ Condition } & Purulent otitis $(\boldsymbol{n}=\mathbf{2 2})$ & Control $(\boldsymbol{n}=\mathbf{3 0})$ & \multirow{2}{*}{$\boldsymbol{p}$} \\
\cline { 3 - 4 } & & Median $[\mathbf{2 5} \mathbf{\%} ; \mathbf{7 5} \mathbf{\%}]$ & Median $[\mathbf{2 5} \mathbf{\%} ; \mathbf{7 5} \mathbf{\%}]$ & $\mathbf{0 . 0 0 0}$ \\
\hline \multirow{2}{*}{ MeanX, mm } & CE & $\mathbf{7 . 0 4}[\mathbf{3 . 3 0} \mathbf{1 6 . 1 6}]$ & $-0.37[-3.13 ; 3.90]$ & 0.213 \\
\multirow{2}{*}{ MeanY, mm } & OE & $-0.72[-5.72 ; 4.47]^{*}$ & $-2.22[-5.70 ; 2.29]$ & $\mathbf{0 . 0 0 0}$ \\
& CE & $-\mathbf{1 3 . 7 2}[-\mathbf{2 5 . 1 7} ;-\mathbf{1 0 . 3 0}]$ & $-4.12[-7.07 ; 0.78]$ & 0.019
\end{tabular}

Note: $C E$ - condition with closed eyes, $O E$ - condition with open eyes, * - significant differences with the values of indicators in the state with closed eyes

Table 2

Characteristics of the shape of the distribution of oscillations of the pressure center in children with purulent otitis media in different sensory states

\begin{tabular}{|c|c|c|c|c|}
\hline \multirow{2}{*}{ Indicators } & \multirow{2}{*}{ Condition } & Purulent otitis $(n=22)$ & Control $(n=30)$ & \multirow{2}{*}{$p$} \\
\hline & & Median $[25 \% ; 75 \%]$ & Median [25\%; $75 \%$ ] & \\
\hline \multirow{2}{*}{ SkewX } & $\mathrm{CE}$ & $-0.03[-0.26 ; 0.10]$ & $-0.01[-0.12 ; 0.15]$ & 0.920 \\
\hline & $\mathrm{OE}$ & $0.04[-0.21 ; 0.23]$ & $0.04[-0.08 ; 0.21]^{*}$ & 0.547 \\
\hline \multirow{2}{*}{ SkewY } & $\mathrm{CE}$ & $0.10[-0.06 ; 0.36]$ & $0.03[-0.09 ; 0.17]$ & 0.227 \\
\hline & $\mathrm{OE}$ & $-0.03[-0.04 ; 0.09]$ & $0.04[-0.15 ; 0.10]^{*}$ & 0.466 \\
\hline \multirow{2}{*}{ KurtosisX } & $\mathrm{CE}$ & $2.74[2.45 ; 2.92]$ & $2.39[2.21 ; 2.71]$ & 0.001 \\
\hline & $\mathrm{OE}$ & $2.42 *[2.29 ; 2.82]$ & $2.55[2.33 ; 2.65]$ & 0.403 \\
\hline \multirow{2}{*}{ KurtosisY } & $\mathrm{CE}$ & $2.58[2.25 ; 2.82]$ & $2.42[2.32 ; 2.55]$ & 0.060 \\
\hline & $\mathrm{OE}$ & $2.57[2.55 ; 2.61]$ & $2.37[2.21 ; 2.65]$ & 0.547 \\
\hline
\end{tabular}

Note: $C E$ - condition with closed eyes, $O E$ - condition with open eyes, * - significant differences with the values of indicators in the state with closed eyes

At the same time, in the presence of visual control, the averaged figure of the projection of the pressure center was observed to elongate along the frontal axis of the averaged figure of the projection of the pressure center of purulent otitis (ratio of $Y / X$ axes $=0.99$ [0.93; 1.59]) (Fig. 2, B). Since postural movements around the frontal axis are mainly controlled by antigravity moments created by dorsal/plantar flexion of the ankle joints, we can assume that in the presence of visual control in children with purulent otitis in the provision of the main posture begins to dominate the ankle strategy. 

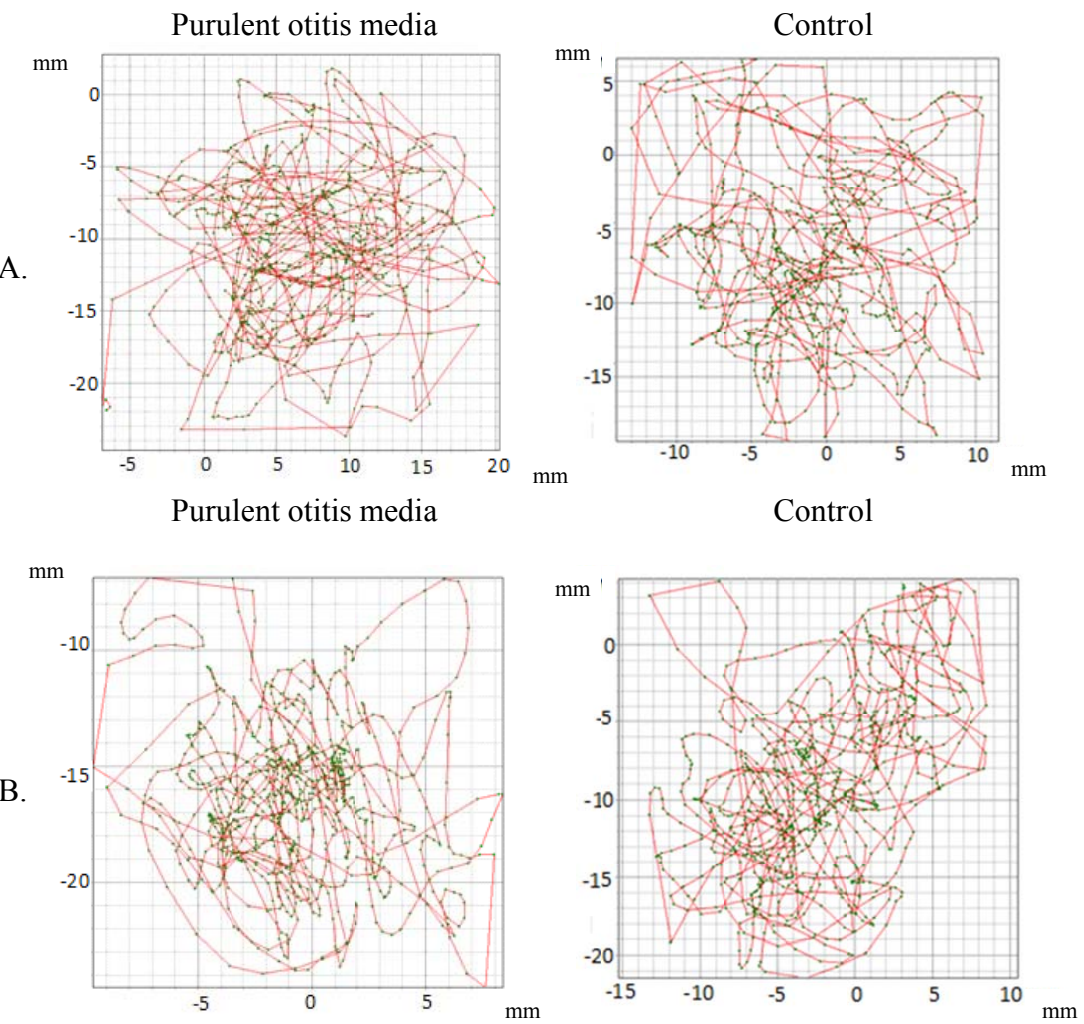

Fig. 2. Examples of stabilograms of children with purulent otitis media and control with closed (A) and open (B) eyes

Analysis of the deviations of the points of the stabilogram allowed us to estimate the stability of the main rack (Table 3). In the state with closed eyes, statistically significant differences between children with purulent otitis media and control of majestic deviations postural oscillations in both the frontal (RangeX, StdDevX) and sagittal (RangeY, StdDevY) plane were not detected, although there was a trend $(p=0.09)$ to increase the amplitude of oscillations in the frontal plane in purulent otitis. However, the involvement of visual afferents in posturographic control led to a statistically significant reduction in deviations postural oscillations only among children with purulent otitis in both the frontal $(p \leq 0.01)$ and sagittal $(p \leq 0.05)$ planes. As a result, children with purulent otitis in the open-eyed condition were characterized by probably $(p \leq 0.05)$ lower amplitude and variability of oscillations of the pressure center, compared with the control, in both planes.

In the state with closed eyes, children with purulent otitis, compared with the control, have significantly $(p \leq 0.001)$ higher values of excess in the frontal plane (Kurtosis $X)$ and a tendency $(p=0.06)$ to higher values in the sagittal plane (KurtosisY) against the same values Pup2Sigma $(p>0.05)$, which indicates a more stable oscillation of the body with fewer large oscillations than in the control. Opening the eyes led to a significant $(p \leq 0.01)$ reduction in excess in the frontal plane in children with purulent otitis, which was not observed in the control. As a result, in the state with open eyes, it was found probably $(p \leq 0.05)$ lower values of Pup2Sigma in purulent otitis compared with the control, which indicates an increase in the number of large oscillations.

A common indicator of the stability of the main posture in stabilometric studies is the angle of oscillation of the pressure center, which in children normally does not exceed $12.5^{\circ}$, which is consistent with the results of our study. It was found that the oscillation angle of the pressure center (\%) in children with purulent otitis is significantly larger $(p \leq 0.001)$ than in the control, in the absence of visual control, while the inclusion of visual afferentation in postural control levelled these differences).

It was found that children with purulent otitis, compared with the control, were characterized by probably faster attenuation of autocorrelation function (Table 4) in both the frontal $(p \leq 0.001)$ and sagittal $(p \leq 0.001)$ planes in the state with closed eyes, while in the state with open eyes faster 
attenuation was observed only in the frontal plane $(p \leq 0.01)$. This indicates less regularity and predictability of oscillatory movements in children with purulent otitis compared with control, ie stabilizing fluctuations were more sudden and/or more pronounced, and probably associated with a higher level of centralization of postural control mechanisms. The latter is consistent with the detected higher average speed of movement of the pressure center $(p \leq 0.001)$ and the greater total length of the statokinesiogram $(p \leq 0.001)$ in children with purulent otitis, compared with the control, in the state with closed eyes.

Table 3

Indicators of the stability of the main rack in children with purulent otitis media in different sensory states

\begin{tabular}{|c|c|c|c|c|}
\hline \multirow{2}{*}{ Indicators } & \multirow{2}{*}{ Condition } & Purulent otitis $(n=22)$ & Control $(n=30)$ & \multirow[b]{2}{*}{$p$} \\
\hline & & Median [25\%; $75 \%$ ] & Median [25 \%; $75 \%$ ] & \\
\hline \multirow{2}{*}{ Length, mm } & $\mathrm{CE}$ & $1445.70[1160.701543 .00]$ & $932.80[769.20 ; 1205.50]$ & 0.000 \\
\hline & $\mathrm{OE}$ & $875.4[789.7 ; 987.4]^{*}$ & $805.70[699.20 ; 933.60]^{*}$ & 0.343 \\
\hline \multirow{2}{*}{ AvgSpeed, $\mathrm{mm} / \mathrm{s}$} & $\mathrm{CE}$ & $19.20[12.76 ; 21.20]$ & $12.04[10.55 ; 16.09]$ & 0.001 \\
\hline & $\mathrm{OE}$ & $10.69[7.90 ; 11.30]^{*}$ & $10.97[9.15 ; 12.69]^{*}$ & 0.343 \\
\hline \multirow{2}{*}{ Angle, ${ }^{\circ}$} & $\mathrm{CE}$ & $6.01[4.49 ; 10.30]$ & $-1.33[-6.59 ; 4.40]$ & 0.001 \\
\hline & $\mathrm{OE}$ & $-0.70[-7.46 ; 8.59]$ & $-4.88[-9.51 ; 4.09]$ & 0.538 \\
\hline \multirow{2}{*}{ Pup2Sigma, \% } & $\mathrm{CE}$ & $91.60[90.00 ; 92.70]$ & $92.00[90.20 ; 95.10]$ & 0.163 \\
\hline & $\mathrm{OE}$ & $90.90[90.00 ; 92.7]$ & $92.45[91.30 ; 93.80]$ & 0.046 \\
\hline \multirow{2}{*}{ RangeX, mm } & $\mathrm{CE}$ & $27.12[21.72 ; 39.31]$ & $25.34[17.88 ; 27.84]$ & 0.090 \\
\hline & $\mathrm{OE}$ & $19.85[17.92 ; 25.45]^{*}$ & $25.83[23.60 ; 29.30]$ & 0.001 \\
\hline \multirow{2}{*}{ RangeY, mm } & $\mathrm{CE}$ & $25.49[23.10 ; 29.05]$ & $25.72[21.80 ; 31.65]$ & 0.693 \\
\hline & $\mathrm{OE}$ & $21.83[17.9 ; 31.52]^{*}$ & $27.32[23.62 ; 31.94]$ & 0.029 \\
\hline \multirow{2}{*}{ StdDevX, mm } & $\mathrm{CE}$ & $5.00[4.49 ; 7.23]$ & $5.10[3.75 ; 6.03]$ & 0.563 \\
\hline & $\mathrm{OE}$ & $4.31[3.49 ; 5.63]^{*}$ & $5.36[4.66 ; 6.14]$ & 0.017 \\
\hline \multirow{2}{*}{ StdDevY, mm } & $\mathrm{CE}$ & $4.95[4.88 ; 7.40]$ & $5.50[4.68 ; 6.44]$ & 0.666 \\
\hline & $\mathrm{OE}$ & $4.77[3.80 ; 6.59]^{*}$ & $5.65[5.13 ; 6.36]$ & 0.083 \\
\hline
\end{tabular}

Note: $C E$ - condition with closed eyes, $O E$ - condition with open eyes, ${ }^{*}$ - significant differences with the values of indicators in the state with closed eyes

Table 4

Spectral-correlation indicators of the stabilogram of children with purulent otitis media in different sensory states

\begin{tabular}{|c|c|c|c|c|}
\hline \multirow{2}{*}{ Indicators } & \multirow{2}{*}{ Condition } & Purulent otitis $(n=22)$ & Control $(n=30)$ & \multirow[b]{2}{*}{$p$} \\
\hline & & Median [25\%; $75 \%$ ] & Median [25\%; $75 \%]$ & \\
\hline \multirow{2}{*}{$\mathrm{CCOX}, \mathrm{c}$} & $\mathrm{CE}$ & $3.31[1.57 ; 5.91]$ & $6.85[4.17 ; 18.19]$ & 0.000 \\
\hline & $\mathrm{OE}$ & $3.70[2.76 ; 7.32]^{*}$ & $4.80[3.78 ; 5.28]^{*}$ & 0.006 \\
\hline \multirow{2}{*}{$\mathrm{CCOY,} \mathrm{c}$} & $\mathrm{CE}$ & $4.00[2.76 ; 7.32]$ & $10.43[6.85 ; 18.90]$ & 0.000 \\
\hline & $\mathrm{OE}$ & $4.41[4.09 ; 6.85]$ & $8.63[5.91 ; 14.33]$ & 0.185 \\
\hline \multirow{2}{*}{ wAvgFX, Hz } & $\mathrm{CE}$ & $0.32[0.27 ; 0.44]$ & $0.29[0.24 ; 0.31]$ & 0.001 \\
\hline & $\mathrm{OE}$ & $0.24[0.24 ; 0.29] *$ & $0.23[0.21 ; 0.28]^{*}$ & 0.000 \\
\hline \multirow{2}{*}{ wAvgFY, $\mathrm{Hz}$} & $\mathrm{CE}$ & $0.34[0.29 ; 0.37]$ & $0.31[0.29 ; 0.37]$ & 0.720 \\
\hline & $\mathrm{OE}$ & $0.26[0.24 ; 0.28]^{*}$ & $0.28[0.25 ; 0.32]^{*}$ & 0.001 \\
\hline \multirow{2}{*}{ PirsonXY } & $\mathrm{CE}$ & $0.11[0.07 ; 0.15]$ & $-0.02[-0.11 ; 0.07]$ & 0.001 \\
\hline & $\mathrm{OE}$ & $-0.02[-0.13 ; 0.17]$ & $-0.08[-0.12 ; 0.09]$ & 0.178 \\
\hline \multirow{2}{*}{ wAvgFXY, Hz } & $\mathrm{CE}$ & $0.31[0.28 ; 0.33]$ & $0.30[0.27 ; 0.34]$ & 0.038 \\
\hline & $\mathrm{OE}$ & $0.24[0.24 ; 0.26]^{*}$ & $0.26[0.23 ; 0.28]$ & 0.848 \\
\hline
\end{tabular}

Note: $C E$ - condition with closed eyes, $O E$ - condition with open eyes, * - significant differences with the values of indicators in the state with closed eyes 
The weighted average frequency of the spectrum of oscillations of the center of pressure in the frontal and sagittal planes and the cross-spectrum in both sensory states was in the low frequency range (about $0.3 \mathrm{~Hz}$ ). This frequency reflects the process of maintaining the center of gravity of the body within the projection of the area of its support and compensatory restoration of the lost balance of the body as a result of the constituent late reflexes. In the state with closed eyes, children with purulent otitis were characterized by significantly higher values of the weighted average frequency of the spectrum of oscillations of the center of pressure in the frontal plane $(p \leq 0.001)$ and its cross-spectrum $(p \leq 0.05)$, compared with controls. Such a shift toward a higher frequency may be a sign of minimal peripheral vestibular dysfunction in children with purulent otitis. At the same time, while maintaining a vertical posture under visual control, children with purulent otitis had probable lower values of the weighted average frequency in the sagittal plane $(p \leq 0.001)$, but higher values in the frontal plane $(p \leq 0.001)$. This may indicate the preservation of signs of minimal peripheral vestibular dysfunction in the regulation of media-lateral balance in children with purulent otitis along with more effective visual-vestibular integration during late control in the anterior-posterior direction under conditions of availability visual afferentation.

Oscillatory movements in the frontal and sagittal planes were independent in both study groups (Table 4), although the degree of their covariance was significantly higher than purulent otitis $(p \leq 0.001)$ in the state with closed eyes. This indicates the dominance of the mechanisms of self-regulation of the main posture in preschool children, in which the intensity of these mechanisms is higher than purulent otitis media.

The results of the estimation of the integral indicator of translational control - the coefficient of the equilibrium function - turned out to be interesting (Fig. 3). In the state with closed eyes, children with purulent otitis significantly $(p \leq 0.001)$ were inferior to the control group in maintaining balance in the main rack.

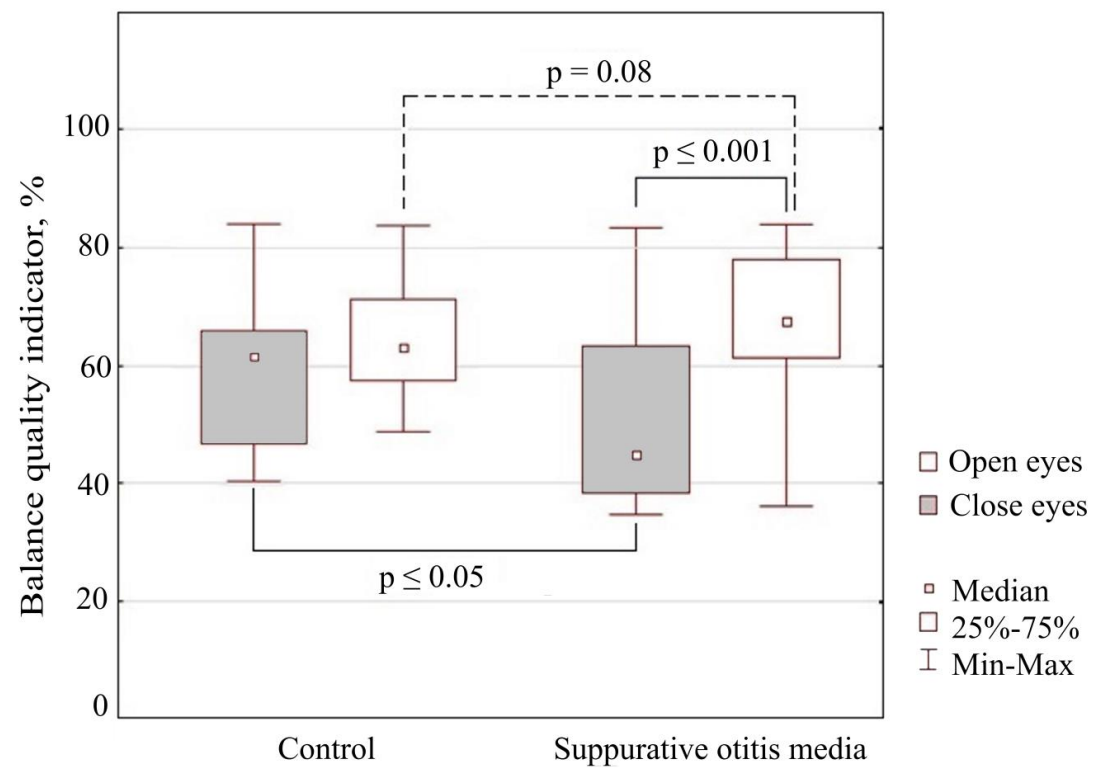

Fig. 3. Indicator of the quality of the balance function in children with purulent otitis media under different sensory conditions

No statistically significant $(p>0.05)$ differences in the integrated indicator of postural control were found between children with different forms of otitis media. However, there was a slight tendency to lower values of the equilibrium quality factor in purulent otitis compared with nonpurulent otitis, especially in the absence of visual control (Fig. 3).

In order to assess the relationship between the visual and proprioceptive systems in the control of balance in the main rack, Romberg coefficients were calculated in children with purulent otitis (Fig. 4). The values of the Romberg coefficient close to $100 \%$ indicate the equality of 
influence of the two equilibrium maintenance systems. In healthy people, the Romberg coefficient by the area of the statokinesiogram varies in the range of $150-300 \%$.

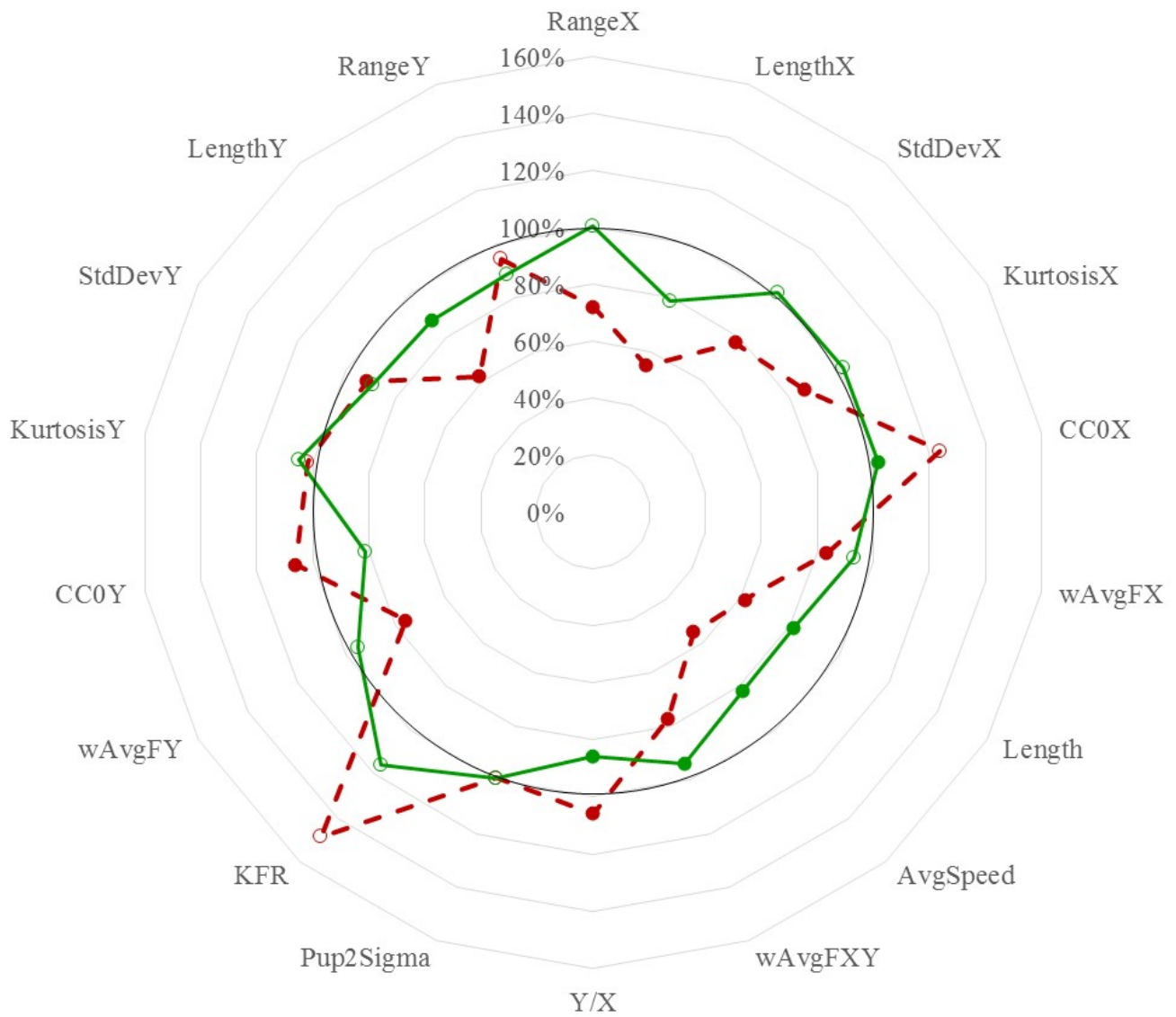

Fig. 4. The value of the average Romberg coefficients (open eyes/closed eyes) in children with purulent otitis: -- $\bigcirc-$ - purulent otitis, $-\bigcirc-$ - control; circles with filling $(\bullet, \bullet)$ denote significant $(p \leq 0.05)$ changes in the values of the indicator in the sample with the eyes open; values higher than $100 \%$ indicate an increase in the values of the indicator, and less than

$100 \%$ - a decrease in the values of the indicator in the sample with the open eyes

It was found that the stabilometric indicators of children with purulent otitis are more reactive to changes in the flow of visual afferents, compared with the control: significant changes were observed in $67 \%$ of the studied indicators, while in the control - only $29 \%$. The amplitude of changes in stabilometric parameters was also higher than purulent otitis. A common feature in both groups was an increase in the stability of the main posture (decrease in Length and AvgSpeed, $p \leq 0.05$ ) in the open-eyed state by strengthening the mechanisms of self-regulation due to the integration of afferent influences from three key systems vestibular, visual and somatosensory wAvgFX, wAvgFY, $p \leq 0.05$ ).

In the control in the sample with open eyes there was a right-hand shift of the amplitude of oscillations in both planes (increase in values), but a significant decrease in predictability and regularity of oscillatory movements was observed in the frontal plane. In contrast, in children with purulent otitis in the sample with eye opening, there was a significant $(p \leq 0.05)$ increase in the quality of balance (increase in KFR) due to the reduction of oscillations (RangeX, RangeY, StdDevX, StdDevY) in both planes and increasing the regularity and predictability of oscillations in the frontal plane. It is likely that visual control of purulent otitis media strengthened the control mechanisms of postural movements around the sagittal axis, which made them more regular and predictable and shifted the coordinates of the pressure center closer to the absolute center in the medio-lateral direction, creating conditions for more effective ankle support. 


\section{Discussion}

Acute otitis media is a fairly common pathological process of the middle ear among preschool children [13, 14]. Previous studies have revealed symptoms of latent vestibular pathology in children with secretory otitis media $[14,18,19,21]$. However, only a small number of these studies focus on a narrow age range - preschool age [16, 17]. We also found no work in the last decade, which would highlight the state of vestibular function in children with purulent otitis media. The variety of vestibular function assessment methods used in the cited studies also attracts attention: from clinical scales of motor development and balance assessment to instrumental research methods (video pulse vestibulo-ocular test (vHIT), electronystagmography (ENG), vestibulo-induced myogenic potentials (oVEMP), stabilometry).

Given the underdevelopment of the problem, the peculiarities of preschool age and the feasibility of using relatively simple objective methods to assess vestibular function in screening studies, we focused on a comparative assessment of vestibular function in children 4-7 years with purulent otitis media by computer static stabilometry.

The main result of our study is to identify the fact of a statistically significant decrease in the stability of the vertical posture in the European position with closed eyes, both in terms of the gold standard in posturography - path length, angle and velocity, and parameters of deviations of the amplitude of oscillations. Violation of the mechanisms of maintaining postural balance in the absence of visual control in children with purulent otitis media is also confirmed by the integrative indicator - the coefficient of quality of the balance function and the coefficients of autocorrelation function. In contrast to other known studies, we also found statistically higher weighted average values of the frequency of the spectrum of oscillations in the frontal and sagittal planes in purulent otitis media compared with control, which may be an additional sign of latent vestibular dysfunction and stress late control mechanisms.

The exact pathogenetic mechanism of vestibular dysfunction in children with otitis media is unknown. In early studies, it was hypothesized that changes in hydrostatic pressure in the middle ear are transmitted through a window and subsequently lead to secondary changes within the fluid of the inner ear [21]. It is also possible that toxins and/or mediators of inflammation from the middle ear can enter the inner ear through the natural membranous barriers and cause inflammation of hair cells [25] and/or damage to the blood-perilymph barrier [26], changing the gradient density between perilymph and endolymph [27]. In particular, the morphological changes of the vestibular neuroepithelium in otitis media are shown [28]. It is possible that altered chemical composition of perilymph and endolymph may affect the ion channels of kinocilli and stereocilia, changing the thresholds of their sensitivity. However, the lower frequency of involvement of vestibular receptors in the pathological process with severe clinical manifestations of vestibular dysfunction, compared with auditory, in otitis media, which according to [2] may be due to low sensitivity of the vestibular neuroepithelium to toxins penetrating into the perilymphatic space from the middle ear, also attracts attention.

Since the maintenance of body balance is the result of the interaction of proprioceptive, vestibular and visual systems, in stabilometric studies to identify the role of each of these systems use modifications of the sensory environment [8]. In our study, we used eye-opening/closing manipulation to separate the contribution of visual afferent and vestibulo-proprioceptive afferent to the postural control of children with purulent otitis.

One of the results of the study was the fact of significantly higher quality of balance function in the main posture in children with purulent otitis compared with the control in the sample with eye opening, which was achieved by reducing the amplitude of postural oscillations, especially in the sagittal plane. It should be noted that according to the results of spectral analysis, this is most likely due to a significant increase in the effectiveness of vestibulo-visual-proprioceptive integration in children with purulent otitis in the regulation of postural oscillations in the posterior-anterior direction against signs of peripheral vestibular dysfunction in support mechanisms.

Taking into account that in children 3-7 years of age, vision has little effect on the parameters of stability, and up to 12-15 years, children are not able to carry out full vestibular-visual 
integration to maintain the stability of posture $[6,29,30]$, we tend to consider the identified phenomenon of better stability of vertical posture in children with purulent otitis media under visual control, as a compensatory mechanism for maintaining balance against the background of latent vestibular dysfunction.

Study limitations. A limitation of this study is that the assessment of vestibular function is based on the assessment of vestibulo-visual-proprioceptive and vestibulo-proprioceptive integration in maintaining static balance, in which the isolated assessment of vestibular and proprioceptive components was not performed. This is primarily due to the involvement in the study of 4-year-old children who are unable to maintain balance on a moving platform for a period of time sufficient for the correct registration of stabilometric indicators. On the other hand, we proceeded from the assumption that the flow of proprioceptive information remains unchanged in states with closed and open eyes. Since the structures of the ear are involved in the pathological process, it was seen that the detected changes in stabilometric parameters in purulent otitis in the state with closed eyes compared to the control will reflect a violation of the vestibular component of vertical posture.

Prospects for further research. Prospects for further research are related to the expansion of the study protocol using dynamic stabilometry. Assessment of vestibular function in purulent otitis media depending on co-variants, such as localization of the pathological process and recurrence rate, as well as assessment of vestibular function in the dynamics of the disease: before treatment, after tympanotomy and in the distant period after recovery $(3,6,12$ months).

\section{Conclusions}

Purulent otitis media in preschool children could lead to latent vestibular dysfunction, which is better detected by static stabilometry in a state with closed eyes. Children 4-7 years with purulent otitis are characterized by higher reactivity of stabilometric indicators to the influence of visual afferentation and better visual-vestibulo-proprioceptive integration while maintaining balance compared to typically developed children, which may be a compensatory mechanism in response to lesions of the vestibular analyzer.

The method of computer static stabilometry could be recommended as a screening method for detecting latent vestibular dysfunction in preschool children, including with otitis media.

\section{Conflicts of interest}

The authors declare that they have no conflicts of interest.

\section{Financing}

The study was performed without financial support.

\section{Acknowledgment}

The authors of the study thank the children who participated in the study and their parents.

\section{References}

[1] Cohen, H. S., Mulavara, A. P., Stitz, J., Sangi-Haghpeykar, H., Williams, S. P., Peters, B. T., Bloomberg, J. J. (2019). Screening for Vestibular Disorders Using the Modified Clinical Test of Sensory Interaction and Balance and Tandem Walking With Eyes Closed. Otology \& Neurotology, 40 (5), 658-665. doi: http://doi.org/10.1097/mao.0000000000002173

[2] Halmágyi, G. M., Curthoys, I. S. (2021). Vestibular contributions to the Romberg test: Testing semicircular canal and otolith function. European Journal of Neurology, 28 (9), 3211-3219. doi: http://doi.org/10.1111/ene.14942

[3] Nishino, L. K., Rocha, G. D., Souza, T. S. A. de, Ribeiro, F. de A. Q., Cóser, P. L. (2021). Protocolo para posturografia estática com provas dinâmicas em indivíduos sem queixas vestibulares utilizando o sistema Horus. CoDAS, 33 (3). doi: http://doi.org/ 10.1590/2317-1782/20202019270

[4] Inojosa, H., Schriefer, D., Klöditz, A., Trentzsch, K., Ziemssen, T. (2020). Balance Testing in Multiple Sclerosis - Improving Neurological Assessment With Static Posturography? Frontiers in Neurology, 11. doi: http://doi.org/10.3389/fneur.2020.00135

[5] Prosperini, L., Castelli, L. (2018). Spotlight on postural control in patients with multiple sclerosis. Degenerative Neurological and Neuromuscular Disease, 8, 25-34. doi: http://doi.org/10.2147/dnnd.s135755 
[6] Orendorz-Frączkowska, K., \& Kubacka, M. (2019). The development of postural control in 6-17 old years healthy children. Part I Postural control evaluation in modified Clinical Test for The Sensory Interaction on Balance in 6-17 old year children (mctsib). Otolaryngologia Polska, 74 (1), 1-7. doi: http://doi.org/10.5604/01.3001.0013.2965

[7] Howcroft, J., Lemaire, E. D., Kofman, J., McIlroy, W. E. (2017). Elderly fall risk prediction using static posturography. PLOS ONE, 12 (2), e0172398. doi: http://doi.org/10.1371/journal.pone.0172398

[8] Gorski, L. P., Silva, A. M. da, Cusin, F. S., Cesaroni, S., Ganança, M. M., Caovilla, H. H. (2019). Body balance at static posturography in vestibular migraine. Brazilian Journal of Otorhinolaryngology, 85 (2), 183-192. doi: http://doi.org/10.1016/ j.bjorl.2017.12.001

[9] Nair, S., Gupta, A., Nilakantan, A., Mittal, R., Dahiya, R., Saini, S. et. al. (2017). Impaired Vestibular Function After Cochlear Implantation in Children: Role of Static Posturography. Indian Journal of Otolaryngology and Head \& Neck Surgery, 69 (2), 252-258. doi: http://doi.org/10.1007/s12070-017-1124-3

[10] Janky, K. L., Givens, D. (2015). Vestibular, Visual Acuity, and Balance Outcomes in Children With Cochlear Implants. Ear \& Hearing, 36 (6), e364-e372. doi: http://doi.org/10.1097/aud.0000000000000194

[11] Monsanto, R. da C., Kasemodel, A. L. P., Tomaz, A., Paparella, M. M., Penido, N. de O. (2018). Current evidence of peripheral vestibular symptoms secondary to otitis media. Annals of Medicine, 50 (5), 391-401. doi: http://doi.org/10.1080/ 07853890.2018.1470665

[12] Sabir, O. A., Johnson, E. G., Hafiz, A. E., Nelson, R. N., Hudlikar, M., Sheth, I., Daher, N. S. (2021). Chronic Effects of Pediatric Ear Infections on Postural Stability. International Journal of Pediatrics, 2021, 1-6. doi: http://doi.org/10.1155/2021/6688991

[13] Xie, C. Y., Chen, D., Liu, F. X. (2021). Factors Related to Tympanic Membrane Perforation in Children with Acute Suppurative Otitis Media. Zhongguo Yi Xue Ke Xue Yuan Xue Bao, 43 (4), 531-535. doi: https://doi.org/10.3881/j.issn.1000-503X.13273

[14] Hariuk, H. I., Pochuieva, T. V., Kulikova, O. O., Lozova, Yu. V. (2021). Main issues of children's incidence of acute otitis media and prospects to solve them. International Medical Journal, 27 (1), 73-76.

[15] Kolkaila, E. A., Emara, A. A., Gabr, T. A. (2015). Vestibular evaluation in children with otitis media with effusion. The Journal of Laryngology \& Otology, 129 (4), 326-336. doi: http://doi.org/10.1017/s0022215115000535

[16] Bista, R., Datta, R., Nilakantan, A., Gupta, A., Singh, A. (2019). Vestibular Dysfunction in Children Suffering from Otitis Media with Effusion: Does Grommet Help? An Observational Study Using Computerized Static Posturography. Indian Journal of Otolaryngology and Head \& Neck Surgery, 71 (4), 537-541. doi: http://doi.org/10.1007/s12070-019-01720-Z

[17] Cohen, M. S., Mandel, E. M., Furman, J. M., Sparto, P. J., Casselbrant, M. L. (2011). Tympanostomy Tube Placement and Vestibular Function in Children. Otolaryngology-Head and Neck Surgery, 145 (4), 666-672. doi: http://doi.org/10.1177/0194599811412038

[18] Rehagen, S. K., Valente, M., Lieu, J. E. C. (2020). Vestibular Screening in Pediatric Patients with Otitis Media. Journal of the American Academy of Audiology, 31 (3), 209-216. doi: http://doi.org/10.3766/jaaa.18101

[19] Tozar, M., Cömert, E., Şencan, Z., Şimşek, G., Muluk, N. B., Kılıç, R. (2020). Video head impulse test in children with otitis media with effusion and dizziness. International Journal of Pediatric Otorhinolaryngology, 129, 109783. doi: http://doi.org/ 10.1016/j.ijporl.2019.109783

[20] Cömert, E., Şencan, Z., Koçak, F. M., Şimşek, G., Muluk, N. B. (2021). Clinical evaluation of the vestibular impairment using video head impulse test In children with acute otitis media. International Journal of Pediatric Otorhinolaryngology, 141, 110568. doi: http://doi.org/10.1016/j.ijporl.2020.110568

[21] Pazdro-Zastawny, K., Zatoński, T. (2020). The effect of middle ear effusion on the inner ear condition in children. Advances in Clinical and Experimental Medicine, 29 (3), 325-330. doi: http://doi.org/10.17219/acem/112601

[22] Pazdro-Zastawny, K., Pośpiech, L., Zatoński, T. (2018). Long-term evaluation of the effect of middle ear effusion on the vestibular system in children. International Journal of Pediatric Otorhinolaryngology, 109, 13-16. doi: http://doi.org/10.1016/ j.ijporl.2018.03.015

[23] Barozzi, S., Socci, M., Soi, D., Di Berardino, F., Fabio, G., Forti, S. et. al. (2014). Reliability of postural control measures in children and young adolescents. European Archives of Oto-Rhino-Laryngology, 271 (7), 2069-2077. doi: http://doi.org/ 10.1007/s00405-014-2930-9

[24] Chen, B., Liu, P., Xiao, F., Liu, Z., Wang, Y. (2021). Review of the Upright Balance Assessment Based on the Force Plate. International Journal of Environmental Research and Public Health, 18 (5), 2696. doi: http://doi.org/10.3390/ ijerph18052696

[25] Aarhus, L., Tambs, K., Hoffman, H. J., Engdahl, B. (2015). Childhood otitis media is associated with dizziness in adulthood: the HUNT cohort study. European Archives of Oto-Rhino-Laryngology, 273 (8), 2047-2054. doi: http://doi.org/10.1007/ s00405-015-3764-9

[26] MacArthur, C. J., Pillers, D.-A. M., Pang, J., Kempton, J. B., Trune, D. R. (2011). Altered expression of middle and inner ear cytokines in mouse otitis media. The Laryngoscope, 121 (2), 365-371. doi: http://doi.org/10.1002/lary.21349 
[27] Kim, C.-H., Lee, J., Choi, B., Shin, J. E. (2021). Nystagmus in adult patients with acute otitis media or otitis media with effusion without dizziness. PLOS ONE, 16 (5), e0250357. doi: http://doi.org/10.1371/journal.pone.0250357

[28] Monsanto, R. da C., Penido, N. de O., Uchiyama, M., Schachern, P., Paparella, M. M., Cureoglu, S. (2020). Quantitative assessment of cochlear and vestibular ganglion neurons in temporal bones with chronic otitis media. European Archives of Oto-Rhino-Laryngology, 278 (2), 331-338. doi: http://doi.org/10.1007/s00405-020-06094-5

[29] Gurov, A. V., Levina, Y. V., Guseva, A. L., Elchueva, Z. G., Efimova, S. P., Gordienko, M. V. (2018). The specific microbiological and clinical features of acute otitis media. Vestnik Otorinolaringologii, 83 (1), 36-39. doi: http://doi.org/10.17116/ otorino201883136-39

[30] Turon-Skrzypinska, A., Uździcki, A., Przybylski, T., Szylinska, A., Marchelek-Myśliwiec, M., Rył, A., Rotter, I. (2020). Assessment of Selected Anthropometric Parameters Influence on Balance Parameters in Children. Medicina, 56 (4), 176. doi: http://doi.org/10.3390/medicina56040176

Received date 12.10.2021

Accepted date 23.11.2021

Published date 30.11.2021
(C) The Author(s) 2021

This is an open access article under the Creative Commons CC BY license

How to cite: Lozova, Iu., Garyuk, G., Pochuieva, T., Redka, I., Samusenko, S. (2021). Evaluation of vestibular function in children with purulent media otitis by computer stabilometry. EUREKA: Health Sciences, 6, 30-42. doi: http://doi.org/10.21303/ 2504-5679.2021.002172 